\title{
A surge of late-occurring meiotic double-strand breaks rescues synapsis abnormalities in spermatocytes of mice with hypomorphic expression of SPO11
}

\author{
Monica Faieta $^{1}$ - Stefano Di Cecca ${ }^{1}$ - Dirk G. de Rooij ${ }^{2,3}$ - Andrea Luchetti ${ }^{4}$. \\ Michela Murdocca $^{4} \cdot$ Monica Di Giacomo $^{5} \cdot$ Sara Di Siena $^{6} \cdot$ Manuela Pellegrini $^{7}$. \\ Pellegrino Rossi ${ }^{1}$ - Marco Barchi ${ }^{1}$
}

Received: 13 April 2015 /Revised: 18 September 2015 / Accepted: 23 September 2015 /Published online: 6 October 2015

(C) The Author(s) 2015. This article is published with open access at Springerlink.com

\begin{abstract}
Meiosis is the biological process that, after a cycle of DNA replication, halves the cellular chromosome complement, leading to the formation of haploid gametes. Haploidization is achieved via two successive rounds of chromosome segregation, meiosis I and II. In mammals, during prophase of meiosis I, homologous chromosomes align and synapse through a recombination-mediated mechanism initiated by the introduction of DNA double-strand breaks (DSBs)
\end{abstract}

This article is part of a Special Issue on "Recent advances in meiotic chromosome structure, recombination and segregation" edited by Marco Barchi, Paula Cohen and Scott Keeney.

Electronic supplementary material The online version of this article (doi:10.1007/s00412-015-0544-7) contains supplementary material, which is available to authorized users.

Marco Barchi

marco.barchi@uniroma2.it

1 Department of Biomedicine and Prevention, Section of Anatomy, University of Rome Tor Vergata, 00133 Rome, Italy

2 Reproductive Biology Group, Division of Developmental Biology, Department of Biology, Faculty of Science, Utrecht University, Utrecht, Netherlands

3 Center for Reproductive Medicine, Academic Medical Center, University of Amsterdam, Meibergdreef 9, 1105 AZ Amsterdam, The Netherlands

4 Department of Biomedicine and Prevention, Section of Genetics, University of Rome Tor Vergata, 00133 Rome, Italy

5 European Molecular Biology Laboratory (EMBL), Monterotondo, Italy

6 DAHFMO, Sapienza University, Rome, Italy

7 Department of Medicine and Health Science "Vincenzo Tiberio", University of Molise, Campobasso, Italy by the SPO11 protein. In male mice, if SPO11 expression and DSB number are reduced below heterozygosity levels, chromosome synapsis is delayed, chromosome tangles form at pachynema, and defective cells are eliminated by apoptosis at epithelial stage IV at a spermatogenesis-specific endpoint. Whether DSB levels produced in Spo $11^{+/-}$spermatocytes represent, or approximate, the threshold level required to guarantee successful homologous chromosome pairing is unknown. Using a mouse model that expresses Spol1 from a bacterial artificial chromosome, within a Spo $11^{-/-}$background, we demonstrate that when SPO11 expression is reduced and DSBs at zygonema are decreased (approximately $40 \%$ below wild-type level), meiotic chromosome pairing is normal. Conversely, DMC1 foci number is increased at pachynema, suggesting that under these experimental conditions, DSBs are likely made with delayed kinetics at zygonema. In addition, we provide evidences that when zygotene-like cells receive enough DSBs before chromosome tangles develop, chromosome synapsis can be completed in most cells, preventing their apoptotic elimination.

Keywords SPO11 $\cdot$ Chromosome synapsis $\cdot$ Double-strand breaks (DSBs) · Meiotic recombination · Meiosis ·

Spermatogenesis

\section{Introduction}

Meiosis is the specialized cellular process through which haploid gametes are formed for sexual reproduction. In mammals, spermatogenesis takes place within the seminiferous tubules, in which mitotic spermatogonia divide, differentiate, and subsequently enter meiotic divisions. During prophase of meiosis I, homologous chromosomes from differing parental origin 
(each consisting of two sister chromatids), pair, and synapse. Following the formation of physical links (chiasmata), they subsequently align and move to opposite poles at metaphase I. At the second meiotic division, sister chromatids separate to form haploid round spermatids, which elongate and eventually mature into spermatozoa (Hamer et al. 2008). Failure of homologous synapsis elicits the activation of a male-specific meiotic checkpoint, which eliminates defective cells via apoptosis, thereby preventing the formation of gametes with damaged or aneuploid genomes (de Rooij and de Boer 2003; Barchi et al. 2005; Hamer et al. 2008; Pacheco et al. 2015).

In mammals, as in many sexually reproducing organisms, proper meiotic chromosome synapsis and the formation of chiasmata depend on the initiation of the recombination process that is induced by the generation of SPO11-mediated double-strand breaks (DSBs) (Keeney 2001, 2008). Subsequent to their formation, DSB ends are repaired by homologous recombination, ultimately giving rise to chromosomal pairing and formation of crossovers ( $\mathrm{CO}$ ), that is, interhomolog DNA links, cytologically identifiable as chiasmata (Keeney 2001, 2008).

During prophase I, the recombination-mediated establishment of stable interaction between homologous chromosomes (henceforth referred to as homologs) is a progressive process. It initiates at leptonema with chromosomal pairing (that is, close juxtaposition between homologs) and proceeds during zygonema, leading to alignment of homologs, whose physical interaction is stabilized by the formation of a zipper-like proteinaceous structure called the synaptonemal complex (SC) (Page and Hawley 2004). In mammals, as in other organisms that rely on recombination for efficient pairing, DSBmediated chromosomal interactions initiate at multiple sites along their length. For this reason, SPO11-mediated DSB numbers largely exceed that of crossovers, and DSBs are made at multiple locations [see (Keeney et al. 2014) and references therein].

In both mice and humans, the Spoll gene encodes two major splice variants, which differ by the inclusion (SPO11 $\beta$ ) or exclusion (SPO11 $\alpha$ ) of exon 2 (Keeney et al. 1999; Romanienko and Camerini-Otero 1999; Bellani et al. 2010). Another splicing event occurs between exons 7 and 8 , with the generation of alternative isoforms of Spo11 $\beta$ and Spol1 $\alpha$ known as bclI and sphI, respectively (Keeney et al. 1999; Romanienko and Camerini-Otero 1999; Bellani et al. 2010). These isoforms are differentially expressed by spermatocytes, during prophase I: SPO $11 \beta$ mRNA peaks in early prophase, when DSBs are made at leptonema and zygonema. SPO $11 \alpha$ appears at zygonema and accumulates in late prophase (pachynema to diplonema stages), when most of the DSBs generated at leptonema have been already repaired and chromosomal pairing has been achieved (Bellani et al. 2010). Complementation of Spo $11^{-/}$by the ectopic expression of SPO $11 \beta^{\text {bclI }}$ under the control of Xmr promoter revealed that, when this splicing isoform is expressed at wild-type levels, it supports proper DSB formation and synapsis of all chromosomes, with the exception of the XY pair (Kauppi et al. 2011, 2013a). In contrast, when SPO11 $\beta^{\text {bcll }}$ expression is reduced relative to that of $S p o 11^{+/-}$mice, proper synapsis of short autosomes and sex chromosomes fails, with the successive formation of chromosome tangles at pachynema (Kauppi et al. 2013a). This finding suggested the existence of an as-of-yet unidentified threshold level of DSBs required to guarantee successful chromosome synapsis (Kauppi et al. 2013a, b). In wild-type mice, an estimated average $\sim 140$ DSBs are made at the onset of recombination process during leptonema (Cole et al. 2012). This number increases (DSB surge) when cells progress to early/midzygonema ( 200 foci per cell), likely in an attempt to enforce the homologous search process (Cole et al. 2012). When Spo11 gene dosage is halved, DSBs are $20 \%$ reduced, at zygonema (Bellani et al. 2010; Cole et al. 2012). Beside this, $\mathrm{Spo}^{+/-}$mice are phenotypically normal (Bellani et al. 2005; Cole et al. 2012; Kauppi et al. 2013a), indicating that in this mouse, SPO11-generated DSB number at zygonema is above the threshold level for proper progression of chromosome paring/synapsis. Whether DSBs produced in Spo $11^{+/-}$mice represent the threshold level for mammalian meiosis is unknown, due to the lack of additional genetic models to further titrate DSB numbers.

In order to develop a genetic tool to delete early meiotic genes, we generated a mouse line in which both SPO11 and the CRE-recombinase were expressed by a bacterial artificial chromosome (BAC), under the Spol1 promoter (Spo11-IRESCre mice) [see Supplemental Fig. 1 and (Pellegrini et al. 2011)]. The analysis of CRE-recombinase activity in vivo revealed that Cre expression (driven by Spoll promoter) was able to delete a post-meiotic gene, recapitulating the phenotype of the knockout mouse model. However, when Spo11IRES-Cre mice were used to delete a gene with an early meiotic function [namely Nbsl (Difilippantonio et al. 2005)], the deletion only partially recapitulated the expected phenotype. From this observation, we reasoned that either the Crerecombinase was correctly expressed but did not reach the critical level needed for $\mathrm{Nbs} 1$ deletion by early stages of meiosis, or Cre expression (and that of SPO11) was delayed/ reduced in early meiosis. According to the latter hypothesis, this mouse model could potentially provide a tool to study effects of changes in SPO11 expression timing or levels, on meiotic chromosome dynamics in vivo.

Using mice expressing the Spo11-IRES-Cre construct on a $S p o 11^{-/-}$background, we have now found that when spermatocytes receive an approximately $40 \%$ reduction in DSB dosage at zygonema, chromosome synapsis progresses normally. In addition, we provided evidence that if cells with critically reduced numbers of DSBs receive a late surge of DSBs, their synaptic abnormalities are resolved, and thus 
spermatocytes progress through prophase I without relevant activation of the prophase I checkpoint.

\section{Results}

\section{Expression of Spo11-IRES-Cre transgene almost fully complements $\operatorname{Spo}^{-/-}$mutation}

To understand whether expression of the Spo11-IRES-Cre transgene is able to complement Spol1 mutation, we generated mice which expressed SPO11 exclusively from a hemizygous Spo11-IRES-Cre transgenic locus, henceforth referred to as $\operatorname{Tg}(\operatorname{Spo} 11)^{+/-}$. Testes from adult mice were analyzed histologically and compared to those of $\mathrm{Spoll}^{-/-}$and $\mathrm{Spo}_{11^{+-}}$ littermates. The latter were used as controls, since this genotype was obtained with higher frequency than wild type and was reported to be phenotypically indistinguishable from

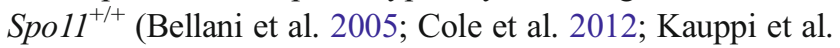
2013a). Weight and size of testis isolated from $\operatorname{Tg}(\operatorname{Spo} 11)^{+/-}$ mice were indistinguishable from that of $S p o 11^{+-}$littermates (Fig. 1a, b). In addition, histological analysis of testis sections revealed that, contrary to Spo11 $1^{-1-}$ (Baudat et al. 2000; Romanienko and Camerini-Otero 2000), in $\operatorname{Tg}(\operatorname{Spo} 11)^{+/-}$ mice, seminiferous tubule composition and cellularity were similar to those in $\mathrm{Spo}_{11}{ }^{+-}$control testes (Fig. 1c). Germ cells progressed through the formation of morphologically mature sperms that did reach the epididymis (Fig. 1c) and the mice were fertile (supplemental Table 1). These observations suggested that the expression of SPO11 driven by the BACderived transgene fully complemented the Spol1 mutation. However, a more detailed analysis of testis cross sections from the above genotypes revealed that a subset of seminiferous tubules of $\operatorname{Tg}(\operatorname{Spo} 11)^{+/-}$mice presented abnormalities.

In mammals, each seminiferous tubule cross section can be assigned to one of 12 epithelial stages (numbered I-XII) based on the array of germ cell developmental stages that it contains (Russell et al. 1990; Ahmed and de Rooij 2009; Muciaccia et al. 2013). Within each epithelial stage, germ cell composition is established by the timely progression of germ cells during differentiation. Thus, the study of germ cell content and composition in each stage is informative in monitoring for defects in germ cell development and loss, over time. The analysis of testis sections from $\operatorname{Tg}(\operatorname{Spo} 11)^{+/-}$mice uncovered that, as shown in Fig. 2a, b and quantified in Fig. 2c, a small but significant percentage $(10.5 \pm 5 \%)$ of seminiferous tubules cross sections missed one or more germ cell generations in at least half of the seminiferous tubule, indicating that germ cell loss had occurred. In male mice, spermatocytes with defects in the timely resolution of DSBs and/or autosomal synapsis are eliminated by apoptosis at epithelial stage IV, which in wildtype spermatocytes corresponds to mid-pachytene stage (de Rooij and de Boer 2003; Barchi et al. 2005; Hamer et al.
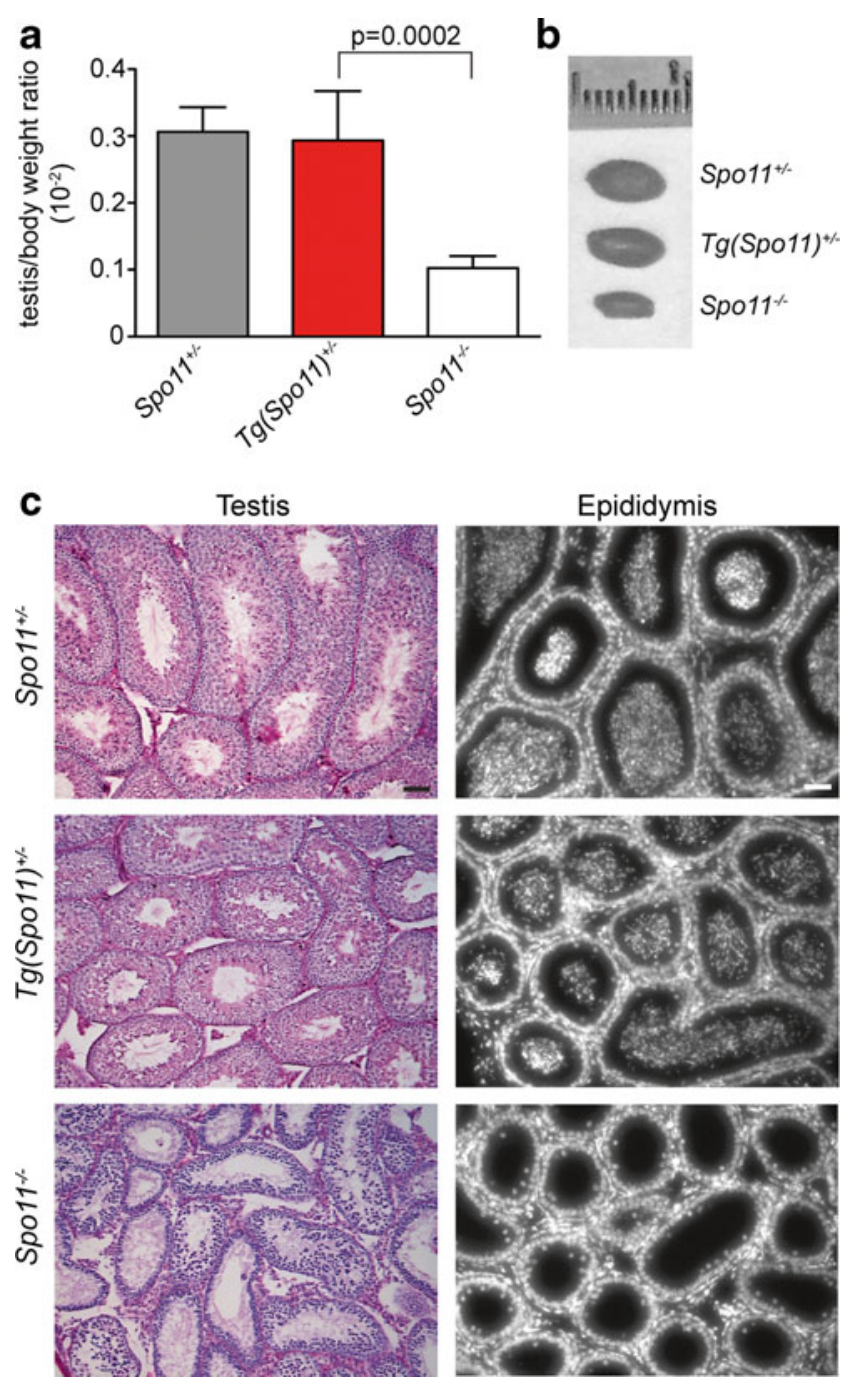

Fig. 1 Histological analysis of testes and epididymis from $S p o 11^{+/-}$, $\operatorname{Tg}(\operatorname{Spo} 11)^{+/-}$, and $S p o 11^{-/-}$mice. a Testis/body weight ratio in adult mice of the indicated genotypes $\left(\operatorname{Spo} 11^{+/-}, n=6\right.$ mice; $\operatorname{Tg}(\operatorname{Spo} 11)^{+/-}, n=$ 9 mice; Spo $11^{-/-}, n=7$ mice); $t$ test $p \leq 0.05$. b Representative image of the testis in the indicated genotypes. $\mathbf{c}$ Periodic acid-Schiff-stained testis cross sections from the indicated genotypes. Hoechst staining of epididymes from mice with the indicated genotypes. Spermatozoa were evident in the epididymis of $\mathrm{Spol1}^{+/-}$and $\operatorname{Tg}(\operatorname{Spo} 11)^{+/-}$mice, while they were absent in Spo $11^{-/-}\left(\right.$Spo $11^{+/-}, n=3$ mice; $\operatorname{Tg}(\operatorname{Spo} 11)^{+/-}, n=5$ mice; $\operatorname{Spol1}^{-/-}, n=3$ mice). Magnification bar represents $50 \mu \mathrm{m}$. Error bars $=$ mean $\pm \mathrm{SD}$

2008; Mahadevaiah et al. 2008; Roig et al. 2010; Pacheco et al. 2015). On the contrary, if cells complete autosomal synapsis but have achiasmatic homolog pairs, or rather fail to synapse the X-Y chromosomes, they undergo apoptosis exclusively at epithelial stage XII, when metaphase I spermatocytes experience the first meiotic division (Eaker et al. 2002; Lipkin et al. 2002; Barchi et al. 2008; Kauppi et al. 2013a). We thus reasoned that if the germ cell loss observed in $\operatorname{Tg}(\operatorname{Spo} 11)^{+-}$tubules was due to both a defect in proper synapsis of the autosomes and segregation at metaphase I, we should have observed a significant increase of apoptosis in both stages IV and XII of the epithelial cell cycle. Our analysis 
a
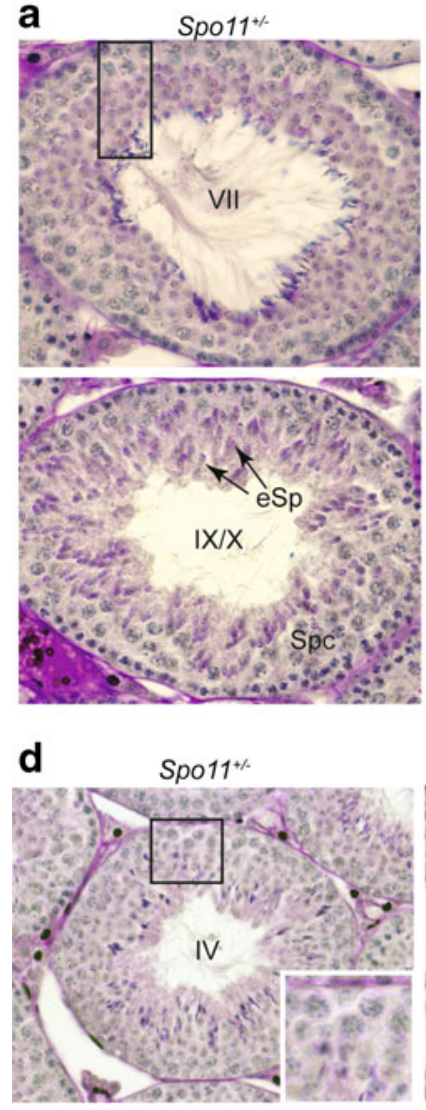
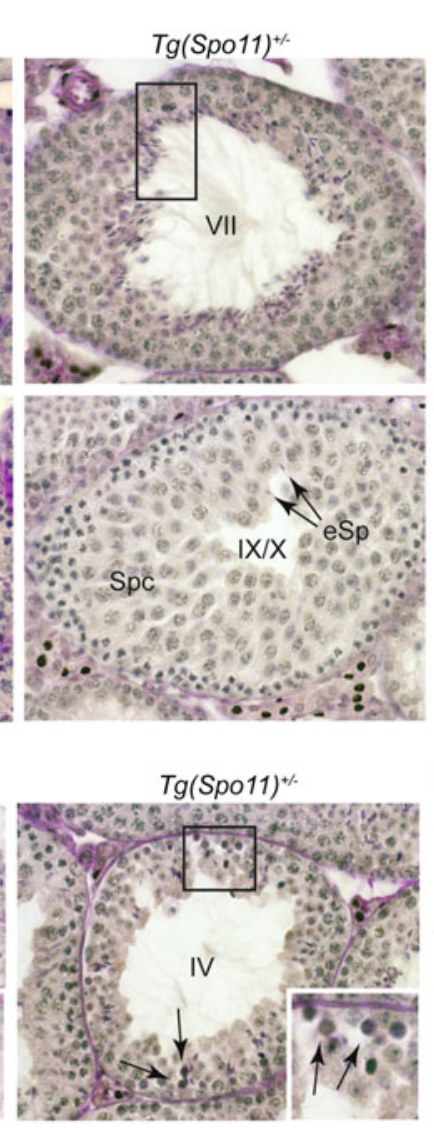

b

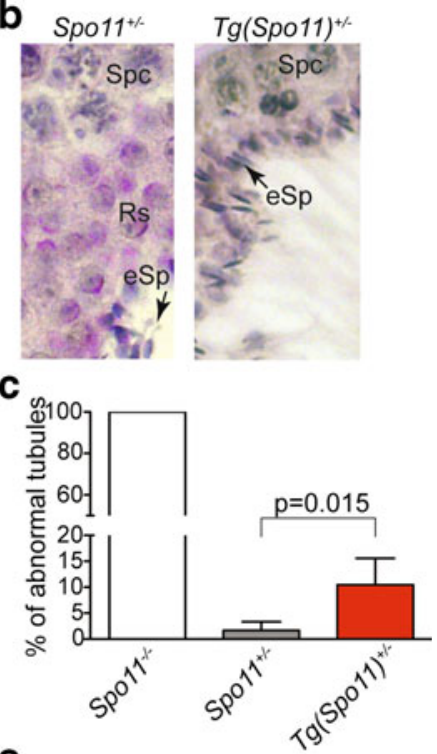

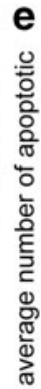

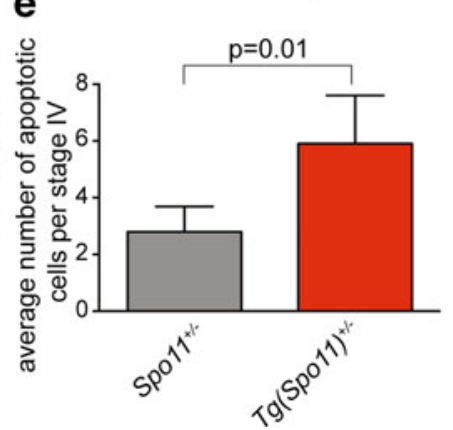

Fig. 2 Analysis of histological abnormalities and apoptosis in testis cross sections of $\operatorname{Tg}(\operatorname{Spo} 11)^{+-}$and control testis. a Periodic acid-Schiff-stained testis sections from the indicated genotypes. Top row: comparison of testis cross sections in stage VII of the epithelial cell cycle. This seminiferous tubule of a $\operatorname{Tg}(\operatorname{Spo} 11)^{+/-}$mouse lacks detectable round spermatids in more than half of the seminiferous tubule cross section, with consequent decrease of cellular density. b Enlargements showing that round spermatids $(R s)$ are always present in the population in $\mathrm{Spoll}^{+-}$, while Rs are missing in about half of this tubule of a $\operatorname{Tg}(\operatorname{Spo} 11)^{+/-}$mouse. $S p c=$ spermatocytes, $e S p=$ elongated spermatids. Bottom row: comparison of testis cross sections in stage IX/X of the epithelial cycle. The tubule from a $\operatorname{Tg}(\operatorname{Spo} 11)^{+/-}$mouse misses $\mathrm{eSp}$.

revealed that in $\operatorname{Tg}(\operatorname{Spo} 11)^{+/-}$mice, there was a significant increase in the proportion of stage IV tubules with at least

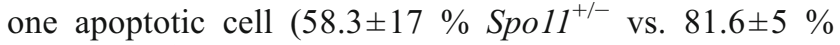
$\operatorname{Tg}(\operatorname{Spo} 11)^{+/-}, p=0.03, t$ test on arcsin-transformed percentages). In addition, as shown in Fig. $2 \mathrm{~d}$ and quantified in Fig. 2e, the average number of apoptotic cells during stage IV increased significantly from $2.8 \pm 0.7$ in $\mathrm{Spo}_{11}^{+/-}$to $5.9 \pm 1.6$ in $\mathrm{Tg}(\mathrm{Spo} 11)^{+/-}$, suggesting that germ cells were lost at the stage IV elimination point (Hamer et al. 2008). In contrast, no significant increase of apoptosis was observed during stage XII (data not shown). Overall, these observations indicated that although spermatogenesis in $\operatorname{Tg}(\operatorname{Spo} 11)^{+/-}$mice is substantially normal, a subset of spermatocytes is eliminated by
Arrows indicate eSp. c Quantification of total amount of tubules at any epithelial stage exhibiting abnormal histology $\left[\operatorname{Spo}_{11^{--}}, n=100\right.$ cross sections, one mouse analyzed; Spol1 ${ }^{+/}, n=499$ cross sections, three mice analyzed; $\operatorname{Tg}(\operatorname{Spo} 11)^{+/-}, n=900$ cross sections, five mice analyzed]. Error bars $=$ mean $\pm \mathrm{SD}$. $T$ test on arcsin-transformed percentages, $p \leq 0.05$. d Periodic acid-Schiff-stained testis sections of tubules at epithelial stage IV. Arrows indicate apoptotic cells. d Quantification of the average number of apoptotic cells per stage IV tubule $\left[\mathrm{Spo} 11^{+/-}, n=110\right.$ stage IV tubule cross sections, $n=3$ mice; $\operatorname{Tg}(\operatorname{Spo} 11)^{+/-}, n=170$ stage tubules cross sections, $n=5$ mice]. Apoptotic cell number was significantly increased ( $t$ test, $p \leq 0.05$ ) relative to the control. Error bars $=$ mean \pm SD

apoptosis, likely due to defects in chromosome synapsis of the autosomes.

\section{$\operatorname{Tg}(\operatorname{Spo} 11)^{+/-}$mice display an overall reduced expression of SPO11}

The establishment of proper synapsis between homologs requires that SPO11 splicing variants (Fig. 3a) are correctly expressed (Kauppi et al. 2011). To understand whether the above-described abnormalities in meiotic progression and apoptosis (Fig. 2) could be linked to the lack of expression of a specific Spo11-splicing isoform from the transgene, we amplified the cDNA from 15 days post-partum (dpp) $\operatorname{Tg}(\operatorname{Spo} 11)^{+-}$ testis using isoform-specific primers. As shown in Fig. 3b, 

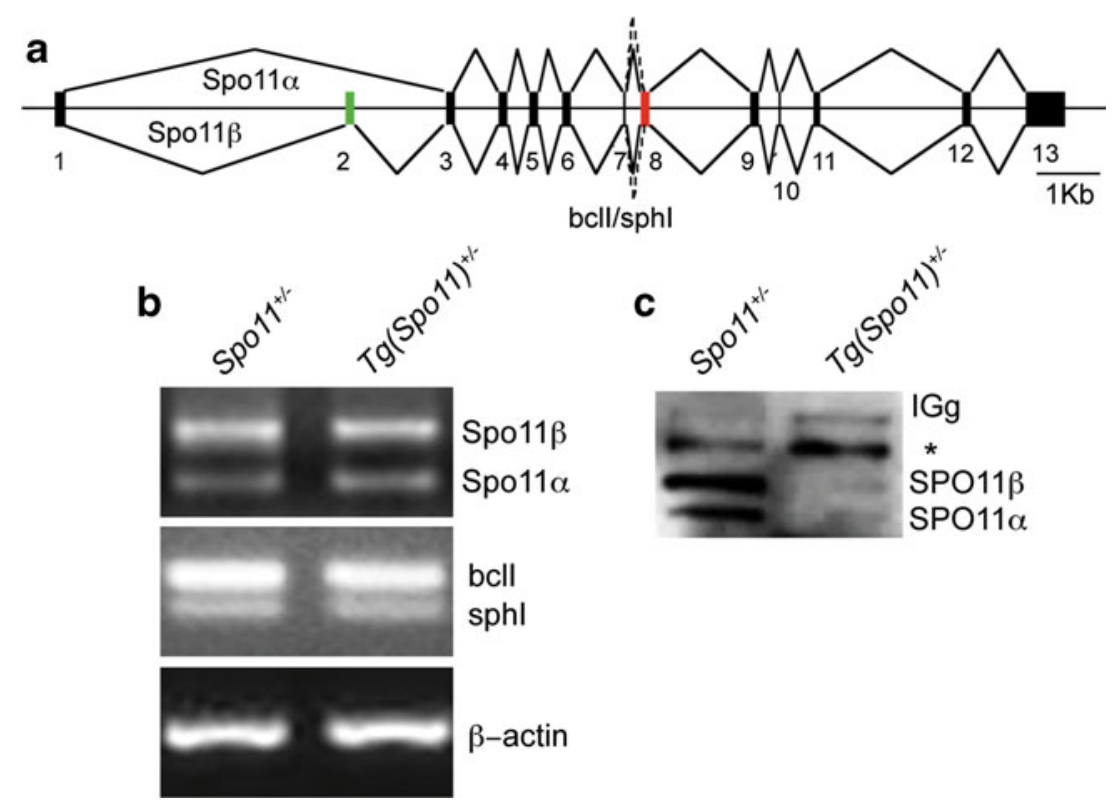

Fig. 3 Expression analysis of Spo 11 splicing isoforms. a Spol1 genomic organization and splicing pattern. Splice variant $\alpha$ excludes exon 2 , whereas variant $\beta$ includes it. The splice junction between exons 7 and 8 varies with respect to the site of the $3^{\prime}$ splice acceptor, including $(b c l l)$ or excluding $(s p h) 12$ nucleotides. b Representative RT-PCR analyses of the expression of Spoll splicing variants in the indicated genotypes. Actin PCR was used as loading control. c Immunoprecipitation of

Spo $11 \beta$ and Spol1 $\alpha$ as well as Spo11-BclI Spol1-SphI transcripts were expressed. In addition, to verify whether the two major isoforms (SPO11 $\beta$ and SPO11 $\alpha$ ) were properly translated, we immunoprecipitated SPO11 from total testis lysate of 45-dpp-old mice. As shown in Fig. 3c, although both isoforms were present, overall protein expression was reduced with respect to $\mathrm{Spo}_{11^{+-}}$control mice. Since in many organisms proper SPO11 expression and DSB number are critical for correct recombination-dependent homologous synapsis (Davis et al. 2001; Tesse et al. 2003; Henderson and Keeney 2004; Kauppi et al. 2013a, b), we next analyzed whether the observed reduction in SPO11 expression in $\operatorname{Tg}(\operatorname{Spo} 11)^{+/-}$mice resulted in any appreciable alteration of chromosome dynamics.

\section{A large fraction of early/mid-zygonema spermatocytes of $\operatorname{Tg}(\operatorname{Spo11})^{+/-}$mice display chromosome synapsis defects}

In mammals, recombination-driven pairing of homologs and synapsis can be monitored by staining meiotic chromosome spreads with antibodies that recognize the axial/lateral element (AE/LE) and central element (CE) of the SC. The SC is a proteinaceous structure that juxtaposes homologous chromosomes in close proximity during the repair of SPO11-mediated DSBs. The SC comprises several proteins, including the AE/ LE protein SYCP3, the assembly of which begins at leptonema. The CE protein SYCP1 assembles along chromosome axes when synapsis of homologous chromosomes

SPO11 in the indicated genotypes. Two independent experiments were performed, one is shown here. The asterisk indicates low-mobility bands likely originating from the Spoll knockout allele expressed in more advanced cell types as demonstrated earlier (e.g., see Kauppi et al. 2013a and supplemental Fig. 2). Note that in $\operatorname{Tg}(\operatorname{Spo} 11)^{+-}$mice, the low-mobility band is stronger than in $S p o 11^{+/-}$as mice carry two copies of the knockout allele

commences (Page and Hawley 2004). In normal spermatocytes at leptonema, axial element formation appears as short stretches of SYCP3 staining. When cells progress to early zygonema, the initiation of chromosome synapsis (visible by the assembly of the SYCP1-positive central element of the SC) occurs simultaneously with the elongation of the axial (now referred to as lateral) element. As cells proceed to mid/ late zygonema, the elongation of chromosome axes is completed, detectable by uninterrupted SYCP3 staining, and homologous chromosome synapsis is achieved over at least $50 \%$ of their length.

Analysis of chromosome synapsis in $\operatorname{Tg}(\operatorname{Spo} 11)^{+/-}$mice revealed that when cells progressed past leptonema, normal synapsing cells (Fig. 4a) appeared concomitantly with cells showing abnormal synaptic features. In contrast, autosomal synapsis was normal in males expressing the Spo11-IRESCre transgene in a $\mathrm{Spo}_{11^{+/+}}$background (data not shown). Hence, it seems likely that $\operatorname{Tg}(\operatorname{Spo} 11)^{+/-}$synaptic defects are tied to the altered expression of the exogenous SPO11 (Fig. 3c), beside other features of the BAC-derived transgene (that is, the expression of Cre-recombinase). In accordance with the uninterrupted extension of the SYCP3-positive axial element and the appearance of (SYCP1-positive) chromosome synapsis, most aberrant-looking nuclei were classified as early/mid-zygotene-like (Em Z-like) cells. These aberrant cells which were morphologically similar to class I cells (Kauppi et al. 2013a) were defined as nuclei with fully elongated SYCP3-positive axes (typical of mid-zygonema cells in 
wild type), with no or very limited overall synapsis (Em Z-like A cells, Figs. $4 \mathrm{~b}$ and $5 \mathrm{c}$ ); or nuclei with more extended overall chromosome synapsis (up to $50 \%$ ), where multiple fully elongated axes remained unsynapsed (Em Z-like B cells, Fig. 4b) but without obvious signs of nonhomologous synapsis. These aberrant cells are in contrast to normal zygotene cells, in which SYCP3-stained axes elongate concomitantly with homologous synapsis progression (Kauppi et al. 2013a). Since Em Z-like cells were never observed in wild type (data not shown), we viewed this as an indication of defects in synaptic progression. The percentage of normal-looking and aberrant nuclei was quantified in 45-93-dpp-old mice by staining of chromosome spreads with SYCP3/SYCP1

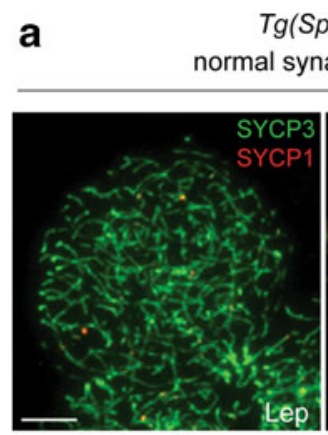

$\operatorname{Tg}(\operatorname{Spo11})^{4-6}$

mal synapsing cells
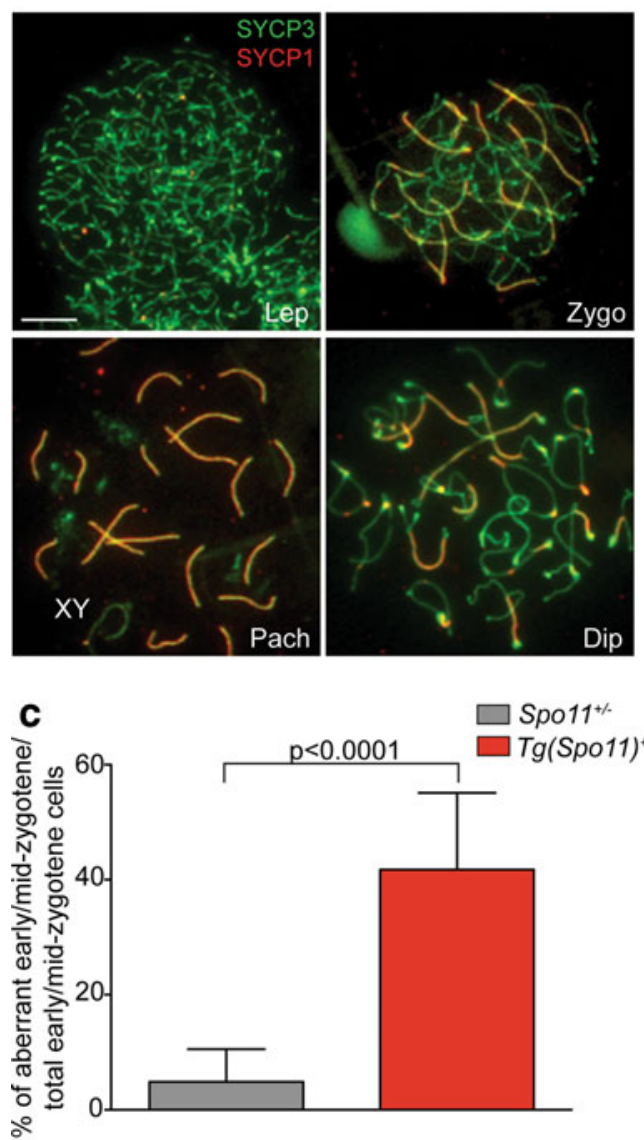

Fig. 4 Analysis of chromosome synapsis abnormalities and meiotic cell progression, in $\operatorname{Tg}(\mathrm{Spo} 11)^{+/-}$and control spermatocytes. Chromosome spreads of $\operatorname{Tg}(\text { Spol1 })^{+/-}$cells immunolabeled for SYCP3 (green) and SYCP1 (red). a Representative images of normal synapsing cells at the indicated stages of prophase I. Lep = leptonema, Zygo = zygonema, $P a c h$ = pachynema, Dip $=$ diplonema. $X Y$ indicates the sex chromosomes. $\mathbf{b}$ Representative images of early/mid-zygotene-like cells of type A ( $E m Z$ like A), type B (Em Z-like B), and of a late zygotene/pachytene-like (Zygo/ Pach-like) cell. The latter cell type was defined as a cell where chromosome synapsis was extended over $50 \%$ and one or more fully elongated (mature) axes (identified by SYCP3 staining) remained completely unsynapsed (white arrow) indicating asynchrony with markers. Surprisingly, in $\operatorname{Tg}(\operatorname{Spo} 11)^{+/-}$mice, a large fraction $(46.3 \pm 10.3 \%)$ of early/mid-zygonema cells appeared abnormal. Among Em Z-like cells, the most

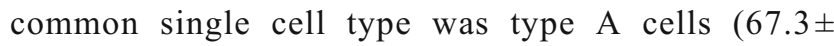
$22 \%$ ). Em Z-like cells were never found in wild-type animals, while they were present at a low percentage in Spo $11^{+/-}$mice (4.8 $\pm 5.4 \%$; Fig. 4c). Remarkably, when (rare) synaptic-defective cells were found at late zygotene/pachytene-like stage, contrary to other mouse models with reduced SPO11 $\beta^{\text {bcll }}$ expression (Kauppi et al. 2011, 2013b), chromosome tangles were rarely apparent (Fig. 4b), suggesting that this phenomenon was somehow prevented.

\section{b $\quad \operatorname{Tg}(\mathrm{Spo11})^{\text {t/ }}$ synapsis defective cells}
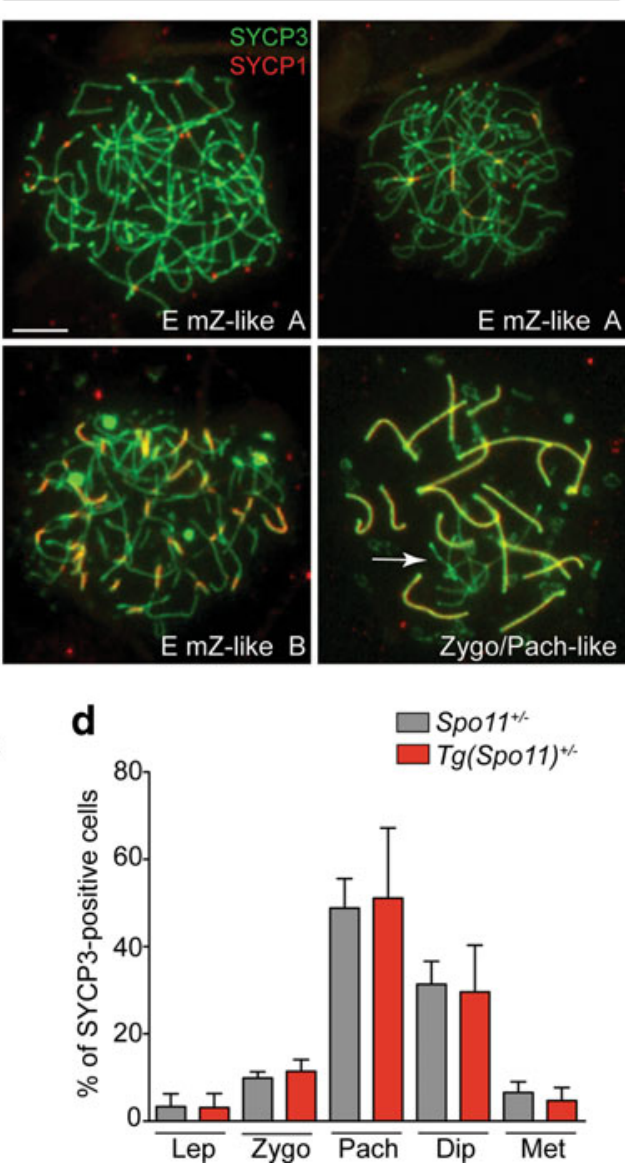

respect to bulk chromosomes. Magnification bar represents $5 \mu \mathrm{m}$. c Quantification of early/mid-zygotene-like cells within the early/midzygonema class, in the indicated genotypes $\left[\operatorname{SpoI1}^{+/-}, n=137\right.$ nuclei analyzed; $\operatorname{Tg}(\operatorname{Spo} 11)^{+/-}, n=196$ nuclei analyzed; six mice tested for each genotype], $t$ test on arcsin-transformed percentages. Error bars $=$ mean \pm SD. d Spermatocytes from 45 to $93 \mathrm{dpp}$ mice of the indicated genotypes were stained for SYCP1/SYCP3 and categorized accordingly to meiotic stage. The zygotene class in $\operatorname{Tg}(\operatorname{Spo} 11)^{+/-}$spermatocytes includes both normally and abnormally synapsing cells $\left[\mathrm{Spo}_{11^{+-}}, n=\right.$ 319 nuclei; $\operatorname{Tg}(\operatorname{Spo} 11)^{+/-}, n=335$ nuclei, three mice tested for each genotype, Bonferroni test, $p=0.7]$. Error bars $=$ mean \pm SD. Met $=$ metaphase I and metaphase II cells 
Spermatogenesis is a continuous process in which spermatocytes mature into spermatids by passing through meiotic sub-stages. If abnormal synapsis of $T g(\operatorname{Spo} 11)^{+/}$spermatocytes remained unresolved, cells die at the stage IV elimination point (de Rooij and de Boer 2003; Barchi et al. 2005; Hamer et al. 2008), with consequent depletion of postzygotene cell types. However, as quantified in Fig. 2e, in $\operatorname{Tg}(\operatorname{Spo} 11)^{+/-}$testis, apoptosis increased only modestly. In addition, the analysis of meiotic cells using prophase I chromosomes stained for SYCP3/SYCP1 markers revealed that the frequency of post-zygotene nuclei of $\operatorname{Tg}(\mathrm{Spo} 11)^{+/-}$mice was not significantly perturbed compared to that of $\mathrm{Spoll}^{+/-}$mice (Fig. 4d), indicating that no significant loss of germ cells at zygonema had occurred. Overall, these observations suggest that most of the cells with aberrant synapsis morphology likely resolved their synaptic problems before reaching the stage IV elimination point and subsequently progressed through prophase I unperturbed.

In mice, as in other species, timely formation, and repair, of an appropriate number of DSBs is critical for proper pairing and synapsis of the homologs (Davis et al. 2001; Tesse et al. 2003; Henderson and Keeney 2004; Kauppi et al. 2013a). To get an insight into the mechanism behind the apparent phenotypic rescue observed in $\operatorname{Tg}(\operatorname{Spo} 11)^{+-}$mice, we next analyzed DSB formation and resolution during prophase I.

\section{Spermatocytes of $\operatorname{Tg}(\operatorname{Spo} 11)^{+/-}$mice receive a reduced/late wave of DSBs}

Timing and levels of DSBs can be studied by staining chromosomal spreads with antibodies against the $\mathrm{AE} / \mathrm{LE}$ element of the SC component SYCP3, and DMC1, a protein known to bind to single strand DNA tails of DSBs (Bishop 1994; Keeney 2001). In agreement with the results above, by analyzing SYCP3-stained spreads of adult spermatocytes, we found that in $\operatorname{Tg}(\operatorname{Spo} 11)^{+/-}$mice, both normal and Em Z-like spermatocytes were present, with the latter being the most abundant $(61 \pm 9 \%, n=322)$ among early/mid-zygotene cells. The analysis of DMC1 foci deposition in $\operatorname{Tg}(\operatorname{Spo} 11)^{+/-}$mice at leptonema, and in normal early/mid-zygotene cells, revealed that consistent with the observed reduction in SPO11 protein level (Fig. 3c), the average number of DMC1 foci was reduced to 33 and $20 \%$, respectively, compared to in $\mathrm{Spoll}^{+/-}$mice, Fig. $5 \mathrm{a}, \mathrm{b}[\sim 58$ and $\sim 40 \%$ of wild-type level (see supplemental Table 2)]. The first semi-synchronous wave in $15 \mathrm{dpp}$ juvenile mice yielded a result comparable with that in adults (Supplemental Fig. 3). However, in $\operatorname{Tg}(\operatorname{Spo} 11)^{+/-}$spermatocytes at early pachynema, the average number of DMC1 foci was significantly increased compared to the controls (Fig. 5a, b), indicating that in these mice, either meiotic DSB repair was delayed or a fraction of pachytene cells developed from zygotene stage cells that had received a delayed wave of DSBs. Since in $\operatorname{Tg}(\operatorname{Spo} 11)^{+/-}$spermatocytes the proficiency of DSBs repair is not expected to be significantly altered with respect to $\mathrm{Spoll}^{+/-}$cells, we favor the latter interpretation.

The quantification of DMC1 foci number in Em Z-like cells revealed that $14 \%$ had no detectable foci. Interestingly, this percentage closely matched that of leptotene stage cells with no foci (12\%), possibly indicating that most Em Z-like cells with no DMC1 foci had never received DSBs (Fig. 5c, d). Nevertheless, the vast majority of Em Z-like cells displayed DMC1 foci, although with highly variable numbers (Fig. 5d). As shown in Fig. 5a, most normal early/midzygonema cells of $\operatorname{Tg}(\mathrm{Spo} 11)^{+/-}$mice displayed a number of DSBs that is higher than the average number of DMC1 foci at leptonema. Because these cells are expected to progress normally through prophase I, such a level of DSBs is likely above the threshold level that supports normal chromosome synapsis (Kauppi et al. 2013a, b). In order to estimate the percentage of Em Z-like cells that receive a surge of DSBs that promote homologous synapsis, we divided Em Z-like cells with foci in two groups: those with DMC1 foci count above and below the average number of foci at leptonema. As shown in Fig. 5c, with this analysis, we estimated that among early/mid-zygotene nuclei, $27 \%$ were Em Z-like cells with an average number of DMC1 foci indistinguishable with respect to that of early/mid-zygotene nuclei with no synaptic defects $(p=0.6$, two-tailed Mann-Whitney test), providing a mechanistic link for their rescue. In the remaining fraction of Em Z-like cells, which account for $21 \%$ of the cells at early/mid-zygonema, the number of DMC1 foci was instead markedly reduced in most cells (Fig. 5d). Because the extension of chromosome synapsis in this latter group was similar, in most spermatocytes, to that of cells with high DMC1 foci count (Fig. 5b and supplemental Fig. 4), one possible interpretation is that Em Z-like cells with high number of $\mathrm{DMC} 1$ foci developed from cells with low foci number, following a rapid surge of DSBs. However, additional mechanisms of rescue cannot be excluded (see "Discussion").

In addition to an appropriate level of DSBs, the rescue of Em Z-like cells would only occur if the DSBs were received before cells undergo apoptosis. To understand if Em Z-like nuclei were apoptotic, we performed a terminal deoxynucleotidyl transferase dUTP nick end labeling (TUNEL) assay on cells stained for DMC1/SYCP3. We focused our analysis on cells with no DMC1 foci, as they were the ones that most likely would be eliminated. We observed that most TUNEL-positive cells were negative for both DMC1 and SYCP3, precluding the analysis (not shown). However, the rare TUNEL-positive spermatocytes where SYCP3-positive axes were still maintained $(n=23)$ lacked DMC1 foci (Fig. 6). Assuming that in TUNEL-positive 
a

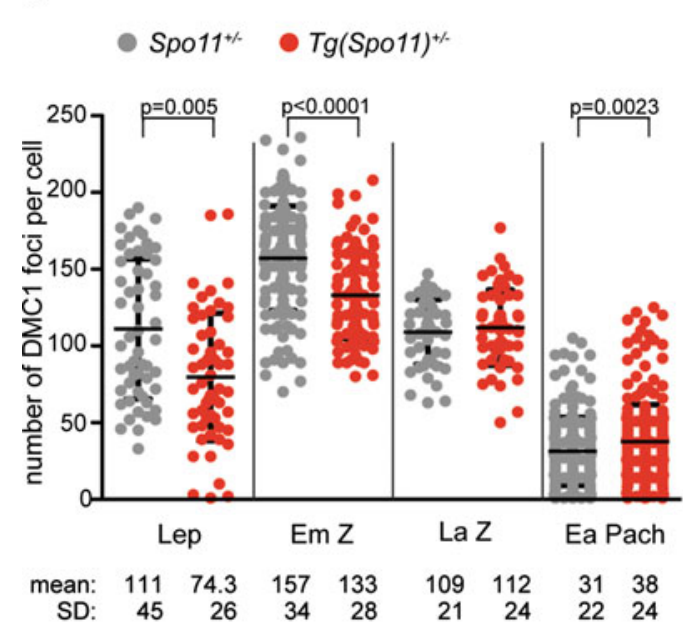

C

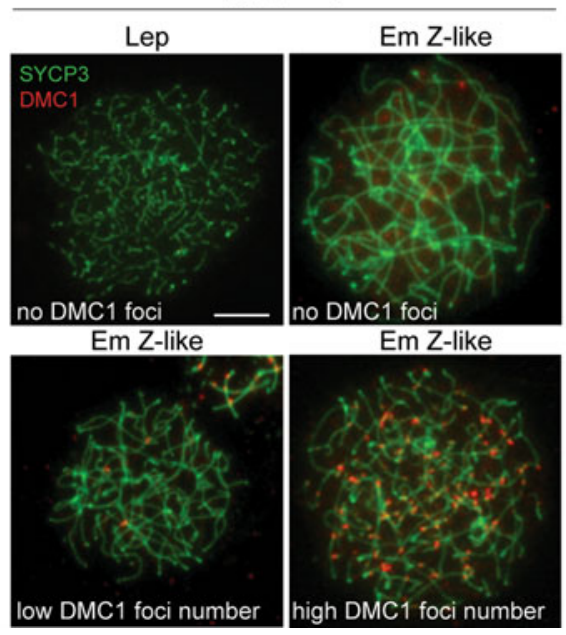

Fig. $5 \mathrm{Tg}(\mathrm{Spo} 11)^{+/-}$spermatocytes undergo a reduced/delayed wave of DSBs. a Quantification of DMC1 foci number in the indicated genotypes. In $\operatorname{Tg}(\operatorname{Spo} 11)^{+/-}$mice, DMC1 foci number is decreased significantly at

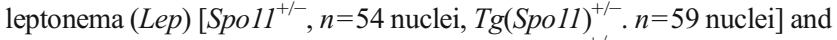
normal early/mid-zygonema $(E m Z)\left[S_{0 o 11^{+/-}}, n=142\right.$ nuclei, $\operatorname{Tg}(\operatorname{Spo} 11)^{+/-}, n=113$ nuclei], while it is increased at early pachytene stage (Ea Pach) [Spol1 ${ }^{+-}, n=209$ nuclei; $\operatorname{Tg}(\text { Spo 11 })^{+/}, n=262$ nuclei]; six mice tested for each genotype. $\mathbf{b}$ Representative nuclear spreads, from the indicated genotypes, labeled with anti-DMC1 and anti-SYCP3. Scale bar $5 \mu \mathrm{m}$. c Representative images of leptotene (Lep) and early/midzygotene-like (Em Z-like) nuclei with no DMC1 foci, and Em Z-like

nuclei, the DMC1 marker is not lost precociously, this observation suggests that the majority of cells in which DSBs were made survived. Interestingly, we also noticed that out of 14 Em Z-like cells with no DMC1 foci and well-preserved SYCP3, none were TUNEL-positive (Fig. 6). This indicates that Em Z-like cells persist for a certain time, before being eliminated.

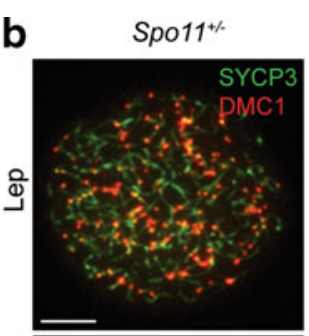

\section{$\operatorname{Tg}(\text { Spo11 })^{+\infty}$}
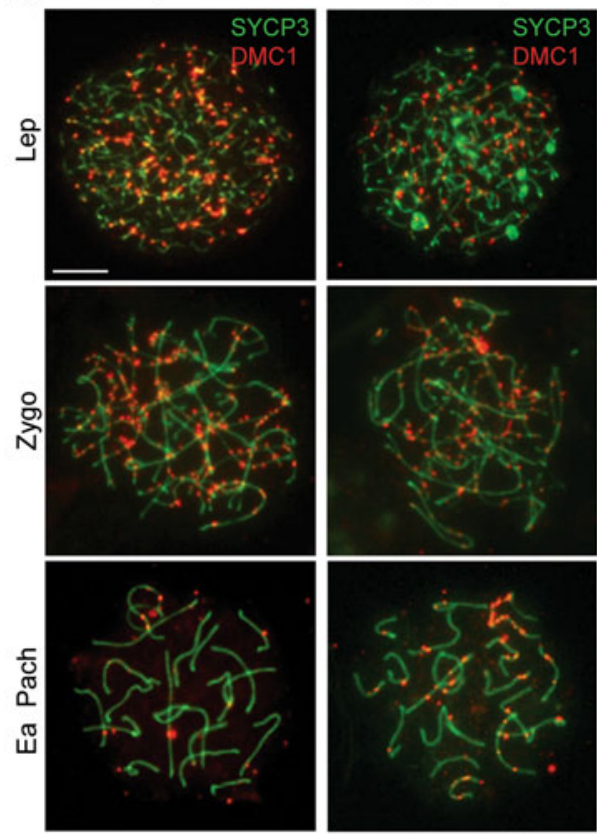

$\operatorname{Tg}(\mathrm{Spo11})^{+\cdots}$

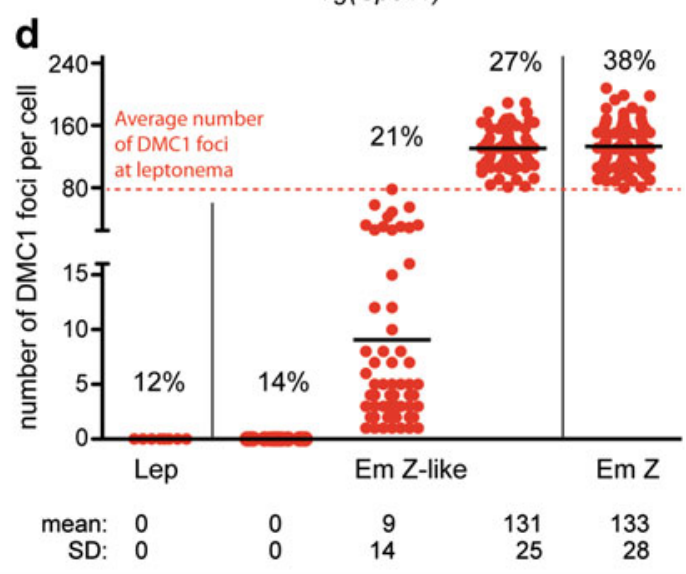

cells with high and low number of DMC1 foci. Scale bar $5 \mu \mathrm{m}$. d Numbers in parentheses are quantification of nuclei with no DMC1 foci at leptotene and early/mid-zygotene-like stages and nuclei with DMC1 foci in normal and aberrant early/mid-zygotene cells of $\operatorname{Tg}(\operatorname{Spo} 11)^{+/-}$ mice (leptotene stage, $n=62$; early/mid-zygotene, $n=209$ ). Each dot in the graph indicates the number of DMC1 foci per nucleus, in normal and Em Z-like cells of $\operatorname{Tg}(\operatorname{Spo} 11)^{+/-}$. The dotted line indicates the average number of DSBs at leptonema, in $\operatorname{Tg}(\text { Spo } 11)^{+/-}$mice. Black bars are means and standard deviations, $p=p$ values (one-tailed Mann-Whitney test)

Overall, these results indicate that normal synapsing cells of $\operatorname{Tg}(\operatorname{Spo} 11)^{+/-}$mice receive, by early/mid-zygonema, a reduced (above threshold level) number of DSBs. However, DSBs do not form timely in all spermatocytes, leading to the formation of Em Z-like cells. The latter last within a time window that precedes their apoptotic elimination and are rescued by a late surge of DSBs. 


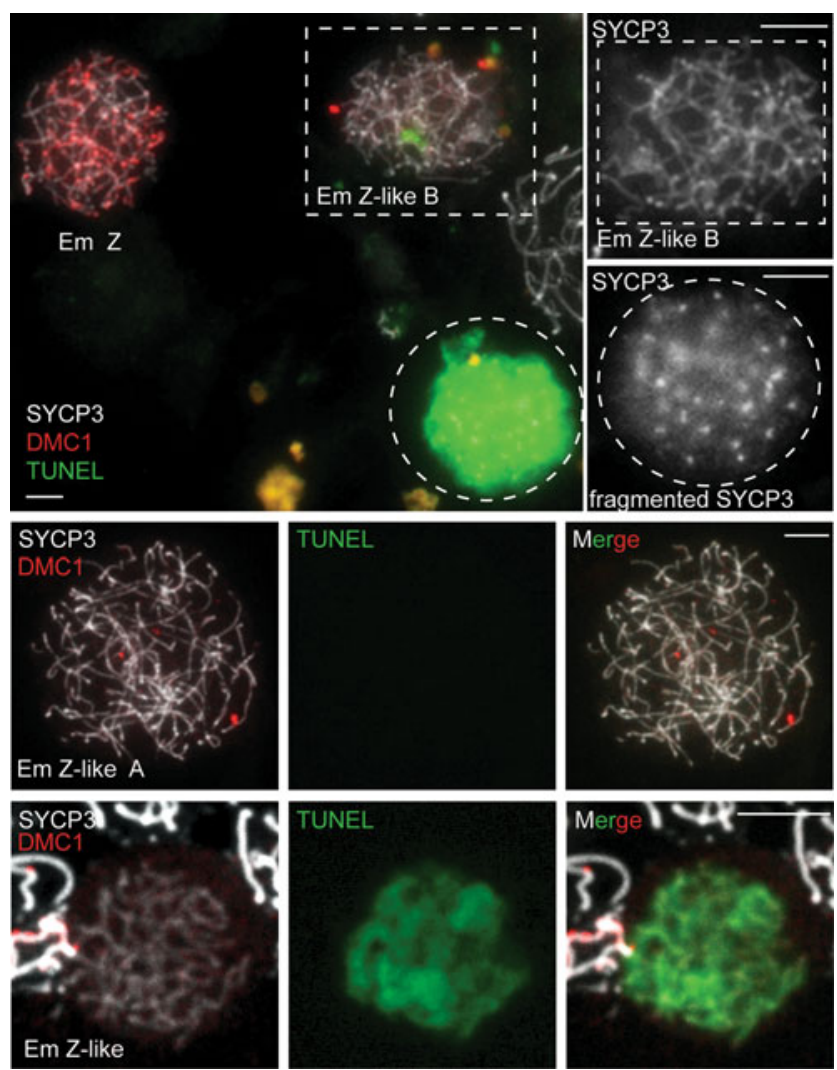

Fig. 6 Em Z-like cells with no DMC1 foci die by apoptosis. Representative images of cells from $\operatorname{Tg}(\operatorname{Spo} 11)^{+/-}$mice, stained with SYCP3, DMC1, and TUNEL. Top row: TUNEL-negative early/midzygotene-like B (Em Z-like B) cell with no foci (highlighted in the dotted square) and a normal early/mid-zygotene $(E \mathrm{mz})$ cell with DMC1 foci, compared to a TUNEL-positive nucleus (Em Z-like B, dotted circle). Images on the right are magnifications of the highlighted cells, showing the morphology of the SYCP3-stained axes. Mid row: representative image of an Em Z-like A cell with no TUNEL signal, indicative of the fact that Em Z-like cells persist before being eliminated. Bottom row: representative image of a rare TUNEL-positive Em Z-like cell with no DMC1 foci, where SYCP3-stained axes appeared still intact. Scale bar $5 \mu \mathrm{m}$

\section{Discussion}

Over recent years, the complementation analysis of the Spo $11^{-/-}$phenotype by different Spo11-expressing transgenes turned out to be an excellent tool for understanding the requirements and dynamics of homologous chromosome pairing in meiosis. The complementation analysis of Spol1 knockout by a transgene that expresses at least wild-type levels of SPO11 $\beta^{\text {bclI }}$ under the control of the $X m r$ promoter has revealed that while the expression of this splicing isoform is sufficient to rescue autosome synapsis, it does not support synapsis of XY chromosomes (Kauppi et al. 2011). In addition to SPO11 $\beta, \mathrm{SPO} 11 \alpha$ is the other main SPO11 splicing isoform (Keeney et al. 1999; Romanienko and CameriniOtero 1999; Bellani et al. 2010); therefore, it has been proposed that $\mathrm{SPO} 11 \alpha$ is required to promote sex chromosome segregation in males (Kauppi et al. 2011). In addition to the above mouse model, it was demonstrated that in mice, as in other species in which recombination is required for stable pairing (e.g., see Terasawa et al. 1995; Lenzi et al. 2005; Storlazzi et al. 2010), the number of SPO11-generated DSBs also matters. Indeed, when DSBs generated by SPO11 $\beta$ were critically reduced with respect to wild type $(\sim 50 \%$ at leptonema, $\sim 75 \%$ at early/mid-zygonema [see supplemental Table 2]) along with XY chromosomes, autosome synapsis also failed, and spermatocytes with entangled chromosomes were eliminated at a pachytene-like stage, by apoptosis (Kauppi et al. 2013a). In contrast, meiotic progression of Spo11 heterozygous mice is completely normal (Bellani et al. 2005; Cole et al. 2012; Kauppi et al. 2013a). Whether

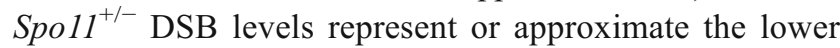
threshold lower limit that can still support chromosome synapsis is yet unknown. In addition, it is worth noting that because in the mouse model of Kauppi et al. (2013a) a reduction in DSB levels was obtained by expressing only the SPO1 $1 \beta^{\text {bcll }}$ isoform, it could not be excluded that the lack of a full suite of Spoll splicing isoforms makes spermatocytes somehow more prone to entanglement of their chromosomes and apoptosis (Kauppi et al. 2013a) (see below).

To study chromosome dynamics in spermatocytes where the ectopic expression of the full set of SPO11 splicing isoforms is not compromised, we complemented Spoll deletion using the Spo11-IRES-Cre transgene. Our analyses revealed that in $\operatorname{Tg}(\operatorname{Spo} 11)^{+-}$mice, overall SPO11 expression was remarkably reduced with respect to $S p o 11^{+/-}$. Nevertheless, male mice were fully fertile, suggesting that the reduced expression of the protein did not significantly impact sperm production. A similar mechanism may operate in females where the same transgene also results in normal fertility. In agreement with this observation, only a small fraction of testis cross sections displayed abnormalities and few spermatocytes died by apoptosis. An unexpected finding was that despite a histologically normal spermatogenesis, the analysis of surface spreads revealed that a substantial percentage of early/midzygotene cells suffered from a delay in chromosome synapsis (Em Z-like cells). During spermatogenesis, timely repair of DSBs and chromosome synapsis are monitored by the prophase I checkpoint. Meiocytes displaying persistent chromosome pairing/synapsis defects are eliminated by apoptosis (de Rooij and de Boer 2003; Barchi et al. 2005; Hamer et al. 2008; Mahadevaiah et al. 2008), with consequent depletion of the germ cell population. The lack of any relevant reduction of post-zygotene cell populations in our model suggested that synapsis abnormalities of most Em Z-like cells were only present transiently and were likely resolved before their possible elimination at the checkpoint.

To gain insight into the mechanism behind this rescue, we analyzed DSB numbers and dynamics, counting DMC1 foci deposition during different meiotic sub-stages. The quantification of DMC1 foci in leptotene spermatocytes from 
$\operatorname{Tg}(\operatorname{Spo} 11)^{+/-}$, revealed that DSB numbers were reduced by $33 \%$ compared to those in $\mathrm{Spo}_{11^{+/-}}$mice $(\sim 58 \%$ with respect to wild type [supplemental Table 2]). In addition, a small percentage of spermatocytes did not have foci (see below). A very similar reduction in DMC1 foci number was previously found in leptotene spermatocytes expressing a reduced dosage of SPO11 $\beta^{\text {bcll }}$ on a Spo $11^{-/-}$background [see (Kauppi et al. 2013a) and supplemental Table 2]. This indicates that in both models, the reduced expression of SPO11 decreases DSB numbers at the onset of meiosis. Quantification of DMC1 foci number in normally synapsing cells of $\operatorname{Tg}(\text { Spo } 11)^{+/-}$spermatocytes at zygonema (Fig. 5a) showed that although the level of DSBs increased during the leptonema to early/mid-zygonema transition, their absolute number was overall reduced in respect to $\mathrm{Spoll}^{+/-}$mice. This observation sets the absolute lower limit of DSB numbers for successful chromosome synapsis at this stage, below that of $\mathrm{Spo}^{+/-}$spermatocytes [approximately in the range of $0.25-0.6$ of the wild-type level (supplemental Table 2)]. The reduction of DMC1 foci number at early/mid-zygonema in $\operatorname{Tg}(\operatorname{Spo} 11)^{+/-}$may be the result of fewer DSBs, consistent with the level of SPO11 protein expression. However, an alternative to reduced absolute levels of DSBs is that spermatocytes have a modified kinetic of DSB formation. In support of this interpretation, $\mathrm{DMC} 1$ foci number at early pachynema were significantly increased compared to those in $\mathrm{Spol1}^{+/-}$. Since DSB formation on unsynapsed axes is a continuous process (Kauppi et al. 2013a), a likely scenario is that during early

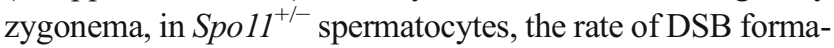
tion overcomes that of repair (surge). When cells progress toward late zygonema, DSB formation rate is reduced, causing a stiff reduction of overall DMC1 foci number. Conversely, in $\operatorname{Tg}(\mathrm{Spo} 11)^{+/-}$cells, the rate of DSBs formation is reduced at leptonema and early zygonema, with respect to $\mathrm{Spol1}^{+/-}$; however, new DSBs continue to be formed by late zygonema with higher frequency than the control and are therefore present in a greater number by early pachynema. Recent evidence implicates ATM kinase in regulating SPO11 DSB formation in mouse spermatocytes through a negative feedback loop (Lange et al. 2011). It is thus likely that, in $\operatorname{Tg}(\operatorname{Spo} 11)^{+/-}$spermatocytes, early reduced DSB formation at leptonema yields a reduced ATM activation and thereby makes cells responsive to mechanisms that are consistent with SPO11 expression levels sustain SPO11 activity at early/mid-zygonema and late zygonema. However, one argument against the hypothesis that in $\operatorname{Tg}(\operatorname{Spo} 11)^{+/}$cells a reduced load of DSBs (and ATM activation) promotes the surge at early/mid-zygonema, is that it does not occur when low levels of SPO11 $\beta^{\text {bcll }}$ are expressed on a Spo $11^{-/}$background (Kauppi et al. 2013a). Because the latter model lacks the full complement of Spol1 splicing isoforms, one possibility is that, in mice, SPO11 activity is subjected to both a negative (ATM-dependent) and positive controls and that the absence of some of the splicing isoforms makes cells unresponsive to mechanisms that positively regulate DSB numbers (surge). Since Mec1 [ataxia telangiectasia-mutated and Rad3-related (ATR) in mammals] has recently been implicated in a positive feedback loop that regulates DSB numbers in yeast (Argunhan et al. 2013; Gray et al. 2013; Cooper et al. 2014), we speculated that SPO11 splicing isoforms (and/or their not yet identified interacting partners) might be targeted by the ATR kinase. If correct, this scenario suggests a multi-layered control of DSB formation in which ATM and ATR, interacting with SPO11 and/or its functional partners, play opposite regulatory roles to guarantee the homeostatic control of DSB formation, under sub-optimal SPO11 expression conditions. Importantly, also in wild-type cells, recombination initiation is a stochastic process, in which at leptonema, meiotic cell receives a widely variable number of DSBs, potentially creating the basis for the establishment of chromosome synapsis defects. Variations in DSB number or kinetics would thus operate also in wild type, on a cell-to-cell basis, depending on the initial load of DSBs and perhaps variability in expression timing of SPO11.

As reported above, along with cells whose chromosome synapsis at zygonema appeared normal, in $\operatorname{Tg}(\operatorname{Spo} 11)^{+/}$spermatocytes, a substantial fraction of early/mid-zygotene cells displayed features of a delay in chromosome synapsis. However, cells with nonhomologous synapsis were rarely apparent, indicating that, as also suggested by others, in mice, nonhomologous synapsis at zygonema is somehow disfavored [see (Kauppi et al. 2013a) and references therein]. Interestingly, however, contrary to what was observed in the model of Kauppi et al. (2013a), such cells never arrested at a pachynema-like stage. Chromosome synapsis defects are thus likely resolved before this arrest point. One possible scenario is that Em Z-like cells of $\operatorname{Tg}(\operatorname{Spo} 11)^{+/-}$mice received a late but "above threshold level" surge of DSBs, reducing the chance that orphan chromosomes (often the $\mathrm{X}$ chromosome) become engaged in nonhomologous interactions [see the model proposed in (Kauppi et al. 2013a)]. Indeed, in support of this interpretation, we found that a relevant fraction of Em Z-like cells ( $\sim 27 \%$ of total early/mid-zygonema nuclei) received a surge of DSBs identical to that of normal-progressing cells at early/mid-zygotene (Fig. 5d). However, not all Em Z-like cells behaved similarly, as a small group of cells ( $\sim 14 \%$ of early/ mid-zygotene stage cells) did not display DMC1 foci. Their percentage almost matched that of leptotene cells with no foci, suggesting that in this subpopulation, SPO11 was either not expressed or expressed at a negligible level, possibly due to a defective expression of the transgene. As indicated by TUNEL assay, these cells likely die by apoptosis at stage IV. In a third group of Em Z-like spermatocytes ( $21 \%$ of cells at early/mid-zygonema), we observed a very wide variation in Dmc1 foci number (from 1 to 79) with most cells displaying 1-15 foci. The latter possibly die or are rescued. The outcome might depend on whether they receive an "above threshold 
level" number of DSBs in a timely fashion, in order to guarantee stable synapsis between the homologs. The small increase in apoptosis observed histologically results from TUNEL assay and the lack of a significant depletion of postzygonema population in $\operatorname{Tg}(\operatorname{Spo} 11)^{+/-}$mice, suggesting that most of these cells will likely complete synapsis before reaching the stage IV elimination point. Intriguingly, recent findings have demonstrated that in yeast, during prophase I, the fate of late-forming DSB changes over time, with lateforming DSBs showing a stronger homologous bias than early ones (Joshi et al. 2015). This is similar to the predicted role, in mice, of SPO $11 \alpha$ in promoting late-forming DSBs, which are more prone to contribute to homologous recognition and segregation of the sex chromosomes (Kauppi et al. 2011). In accordance with these findings, in addition to the formation of a set number of DSBs that overcomes the threshold level, possibly, in some Em Z-like cells, relatively low numbers of DSBs are made late and are more prone to contribute to correct chromosome synapsis, thus being sufficient to rescue cells before the apoptotic elimination point. In this perspective, one could imagine that the threshold would tend to decline over time. The outcome, however, might depend on the overall status of synapsis at the time when late-forming DSBs are made, with cells with more advanced synapsis (that is, Em Z-like type B cells), being perhaps more prone to be rescued by this latter mechanism.

In conclusion, in $\operatorname{Tg}(\operatorname{Spo} 11)^{+/-}$mice, cells are able to cope with reduced (below heterozygosity) levels of DSBs. Likely, this is due to the presence of the full set of Spoll splicing isoforms that, even when SPO11 expression was reduced, allow cells to still receive a surge of DSBs at zygonema that overcome the threshold level, promoting proper homologous search. However, when cells at leptonema and early/midzygonema receive too few DSBs, chromosome synapsis initiation is delayed and cells reach an Em Z-like stage without evident signs of nonhomologous synapsis. These cells persist within a time window where nonhomologous synapsis is prevented, and they are competent to receive a late surge of DSBs. If late-forming DSBs are above "threshold level" spermatocytes, synapsis progresses further and is completed successfully, leading to an overall normal progression of cells through the successive phases of spermatogenesis (summarized in Fig. 7).

\section{Material and methods}

\section{Generation of $\operatorname{Tg}(\operatorname{Spo} 11)^{+/-}$mice and transgene copy number}

Spo11 ${ }^{-/-}[$TgSpol1-IRES-Cre $]$mice, named $\operatorname{Tg}(\operatorname{Spo} 11)^{+/-}$, were obtained at first, by crossing mice carrying TgSpol1IRES-Cre transgene in a wild-type background (Pellegrini et al. 2011), with $S p o 11^{+/-}$mice (Baudat et al. 2000). Then, the resulting Spo11 ${ }^{+/-}[\mathrm{TgSpo} 11$-IRES-Cre $]$ offspring was crossed again with $\mathrm{Spo}_{11^{+-}}$mates. All mice were maintained in a C57/BL6 X 129S/v mix background. To minimize variability from strain background, experimental animals were compared to controls from the same litter or from the same mating involving closely related parents. Each analysis was done with at least three animals.

In order to quantify $\operatorname{Tg}($ Spoll-IRES-Cre) transgene copy number in $\operatorname{Tg}\left(\mathrm{Spo}_{11}\right)^{+/-}$mice, we performed a real-time PCR assay, based on evaluation of genomic Spoll sequence from the transgene. Probe and primers were specific for exon 5 of the Spoll gene, replaced by the Neo cassette in the knockout allele (Baudat et al. 2000). Multiple generations from $\operatorname{Tg}(\operatorname{Spo} 11)^{+/-}$mice $(n=4)$ were tested to exclude any recombination event and changes in transgene copy number through successive generation. All transgenic mice analyzed resulted to be in concordance with Spo11 wild-type copy number (two copies), used as relative control, while one copy was obtained from $\mathrm{Spol1}^{+/-}$, as expected. No Spoll sequence amplification was observed with DNA from Spol1 ${ }^{-/-}$mice (negative control).

$\operatorname{Tg}(\operatorname{Spo} 11)^{+/-}$mice generated close to $50 \%$ of transgenic and $50 \%$ of non-transgenic progeny and copy number were similar among transgenic littermates, consistent with a single stable site of Spo11-IRES-Cre BAC transgene integration in the founder. Thus, $\operatorname{Tg}(\operatorname{Spo} 11)^{+/-}$ are hemizygous for Spo11-IRES-Cre transgene integrated in two copies array at a single stable site. Real-time PCR was performed using a TaqMan-based assay (Life Technologies) with Taqman Universal master mix II (Life Technologies). The comparative $\Delta \Delta \mathrm{Ct}$ method was used to quantify relative gene expression. Primers and probes for Spoll gene were designed as follows: Spol1 probe 5'-6 FAM-TATGCTGAAAGTGCCCAGGMGB-3'; primers: $S p o 11$ sense $5^{\prime}$ CGCCATCGATGACATTTCC-3'; and Spoll antisense 5'-GGCTCACCACGTGCAGACT-3'. The expression of mouse $A p o B$ was used as reference gene; $A p o B$ Probe VIC 5'-CCA ATG GTC GGG CAC TGC TCA A-3' MGB, Primers ApoB sense 5'-CAC GTG GGC TCC AGC ATT-3', and ApoB antisense $5^{\prime}$ TCACCAGTCATTTCTGCCTTT-3'. All primers and probes for real-time PCR assay were designed with Primer Express Software v3.0.1 (Life Technologies, Applied Biosystems).

\section{Genotyping}

Genotyping was performed by both conventional and realtime PCR of tail tip DNA. Genotyping of Cre sequence was executed with conventional PCR by using the following set of primers: Cre sense 5'-AAT GCT TCT GTC CGT TTG CCG 


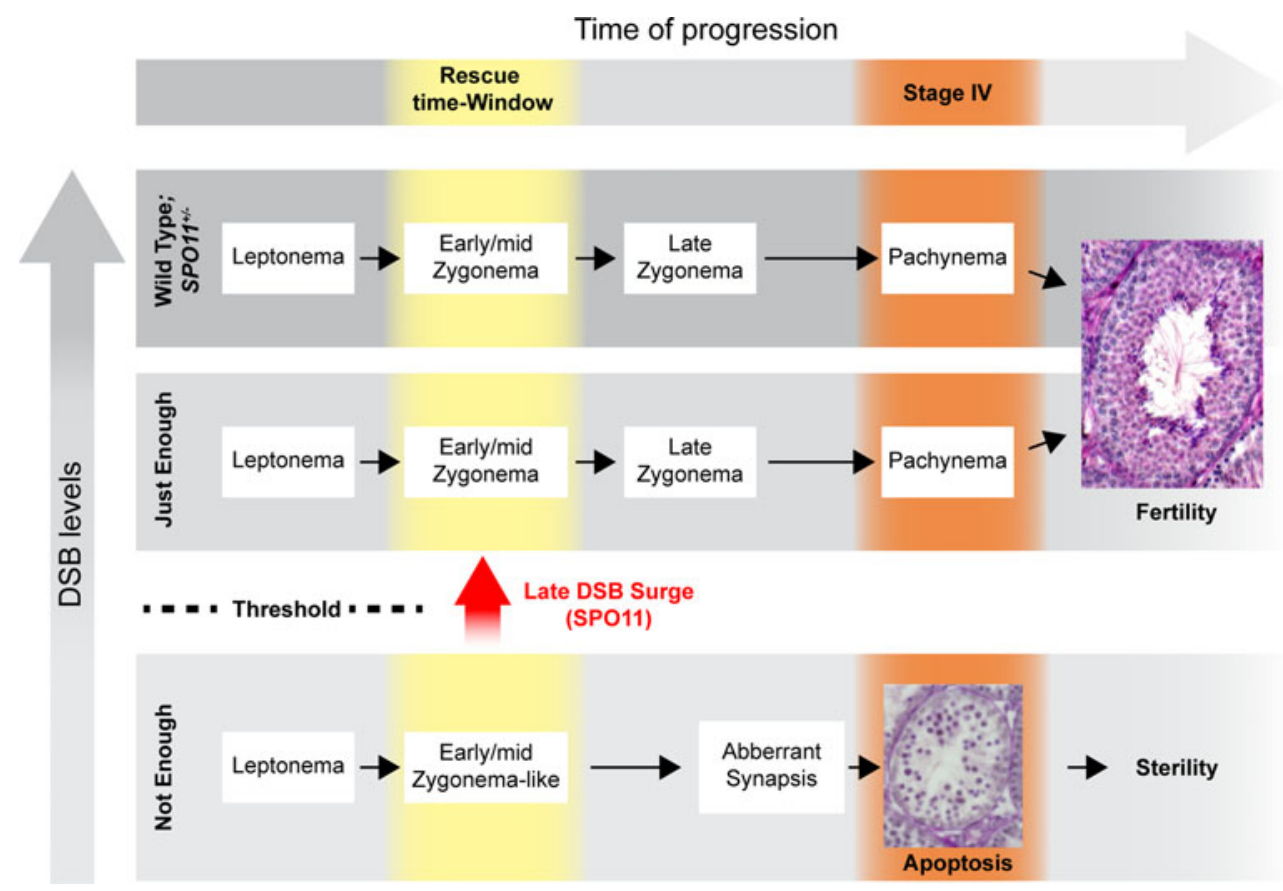

Fig. 7 Model summarizing the effect of DSB reduction and late DSB formation on spermatocyte meiotic progression. In wild-type and Spo $11^{+/}$ mice, double-strand break (DSB) levels support proper chromosome synapsis and meiotic progression (top gray horizontal bar) and spermatocyte progress through prophase I, up to the formation of mature sperms. If DSBs are reduced below the level of Spo $11^{+/-}$, but above the threshold (just enough), spermatogenesis is successful (mid gray horizontal bar). However, if at leptonema and early/midzygonema, "not enough" DSBs are generated (bottom gray horizontal bar), chromosome synapsis is delayed, and early/mid-zygotene-like spermatocytes appear. If no (or below-threshold levels) DSBs are further generated, spermatocytes undergo nonhomologous synapsis, arrest at a pachytene-like stage, and die by apoptosis at stage IV of the epithelial cell cycle (Kauppi et al. 2013a). In contrast, if early/midzygotene-like cells receive a late surge of DSBs before chromosomes entangle and start to die (rescue time window), chromosome synapsis is rescued and cells progress through prophase I, up to the formation of mature sperms

\section{G-3' and Cre antisense 5'-CCA GGC TAA GTG CCT TCT} CTAC-3'. Genotyping of Spoll endogenous allele was performed by conventional PCR by using the following primers: Sp1F sense 5'-CTA CCT AGA TTC TGG TCT AAG C-3', PRSF2 5'-CTG AGC CCA GAA AGC GAA GGA A-3', and Sp16R 5'-ATG TTA GTC GGC ACA GCA GTA G-3' (Baudat et al. 2000). Since PCR primers Sp1F/Sp16R also recognize the Spoll wild-type allele from the BAC vector, to identify mice carrying the transgene within an endogenous knockout genetic background $\left[\operatorname{Tg}(\operatorname{Spo} 11)^{+/-}\right.$mice], we determined the Neomicine (Neo) cassette copy number from the knockout allele (Baudat et al. 2000). Indeed, the presence of two copies of the Neo cassette identified the Spol1 ${ }^{-/-}$mice, implying that Spoll conventional PCR positivity was due to the transgenic copy. To quantitate Neo copy number, we used a TaqMan Real-Time PCR (Life Technologies) assay, performed as previously described and $A p o B$ was used as reference gene. Primers and probes were designed with Primer Express Software v3.0.1 (Life Technologies, Applied Biosystems): Neo Probe FAM 5'TGGCCGCTTTTCT-3' MGB and primers Neo sense 5'-TGC CGA ATA TCA TGG TGG AA-3' and Neo antisense 5'-GAT TCA TCG ACT GTG GCCG-3'.

\section{Tissue collection and histological analyses}

Testes and epididymis were collected and fixed overnight at $4{ }^{\circ} \mathrm{C}$ in Bouin's fixative (Sigma no. HT10132). Freshly collected testes were weighted before fixation. Mean of testis weights was calculated and normalized on body weight to minimize difference among animals. Fixed and paraffinembedded testes were sectioned at $5 \mu \mathrm{m}$ and stained with periodic acid-Schiff/hematoxylin. For histological evaluation of tubule abnormalities and cell death, cross sections were collected at $100 \mu \mathrm{m}$ distance. Staging of spermatogenesis was performed according to the method of Russell et al. (1990) and Ahmed and de Rooij (2009). Paraffin-embedded epididymes were stained with Hoechst. Coverslips were mounted using ProLong ${ }^{\circledR}$ Gold Antifade Mountant (Molecular Probes no. P36934).

\section{Surface chromosome spread preparation and immunofluorescence}

Prophase I chromosome preparations and immunofluorescence were performed using previously described techniques (Barchi et al. 2005, 2009). In brief, testes were removed from euthanized animal, decapsulated, chopped in MEM-HG, and 
mixed. Suspension was let to settle down and supernatant was spun down at 7,500 rpm for $1 \mathrm{~min}$. The pellet was resuspended in $0.5 \mathrm{M}$ sucrose. The suspension was added to slides coated with PFA $1 \%$ and $0.015 \%$ TX-100 and incubated for $2 \mathrm{~h}$ in a humidified chamber at room temperature. At the end of incubation, slides were rinsed twice in 1:250 Photo-flo Kodak professional (no. 1464510) in water and allowed to air dry. Surface chromosome spreads were either immediately processed for immunofluorescence or stored at $-80^{\circ} \mathrm{C}$ for up to 6 months. For immunofluorescence, surface chromosome spread preparations were incubated overnight at room temperature with the primary antibody diluted in antibody dilution buffer (ADB, $10 \%$ goat serum, $3 \%$ bovine serum albumin [BSA], $0.05 \%$ Triton X-100 in phosphate-buffered saline [PBS]). At the end of the incubation, slides were washed once in washing buffer $1(0.4 \%$ Photo-flo, $0.01 \%$ Triton X-100 in water) and once in washing buffer $2(0.4 \%$ Kodak Photo-flo in water) for $10 \mathrm{~min}$. Slides were incubated with the secondary antibody for $1 \mathrm{~h}$ in a pre-warmed humidified chamber at $37^{\circ} \mathrm{C}$ in the dark. After 10-min washes with washing buffers 1 and 2 , slides were immersed for 1 min into $1 \times$ PBS and dried at room temperature in the dark. Coverslips were mounted using ProLong ${ }^{\circledR}$ Gold Antifade Mountant with DAPI (Molecular Probes no. P36935). Images were captured using Leica CTR6000 Digital Inverted Microscope connected to a charge-coupled device camera and analyzed using the Leica software LAS-AF, for fluorescent microscopy. Sources and dilutions of antibodies for immunofluorescence were as follows: mouse anti-SYCP3 (Santa Cruz sc-74569) 1:200, rabbit anti-SYCP1 (Abcam ab15090) 1:200, and rabbit anti-DMC1 (Santacruz no. sc-22768) 1:100. The specificity of anti-DMC1 antibody was verified by staining chromosome spread preparation from $\mathrm{Dmcl}^{-/-}$mice. Secondary antibodies used were goat anti-mouse Alexa 488 (no. A1101), goat anti-rabbit Alexa 594 (no. A11012), and goat anti-mouse Alexa 647 (no. 150115) all 1:200, Invitrogen. TUNEL assay was performed according to the method of Pacheco et al. (2015) using the In Situ Cell Death Detection Kit (POD) from Roche.

\section{RNA extraction and RT-PCR}

For RT-PCR, 15-dpp-old mice testes from $\mathrm{Spol1}^{+/-}$and $\operatorname{Tg}(\operatorname{Spo} 11)^{+-}$mice were extracted using reagent QIAzol lysis reagent (Qiagen no. 79306). Contaminating genome DNA was removed with DNA-free kit (Ambion no. AM1906). cDNA synthesis was performed with Invitrogen RT-PCR SuperScript III (Invitrogen, no. 18080-051). Spo11 $\beta$ and Spo $11 \alpha$ transcripts were amplified with the following primers: Spo $11 \beta / \alpha 2$ F 5'-GTT GGC CAT GGT GAA GAG AG-3'; and Spo $11 \beta / \alpha$ R3 5'-TTT TGG TGA ATC GCT TCT GA-3'. Spoll Bcll and SphI isoforms were amplified with the following primers: Spo $11 \mathrm{~b} / \mathrm{s} F 5^{\prime}$ -
GCCGACTAACATTCAAGGAATGC-3' and Spo11 b/s R 5'-TGCAGAAGTTGTCGTCCAGGAG-3'.

\section{SPO11 immunoprecipitation and western blot}

Immunoprecipitation (IP) was performed using previously described techniques (Neale et al. 2005; Lange et al. 2011). In brief, testes from 45-dpp Spo $11^{+/-}, \mathrm{Tg}(\operatorname{Spo} 11)^{+/}$, and Spo $11^{-/}$ - mice were decapsulated and lysed in $800 \mu$ lysis buffer $(1 \%$ Triton X-100, $400 \mathrm{mM} \mathrm{NaCl}, 25 \mathrm{mM}$ HEPES-NaOH at $\mathrm{pH}$ 7.4, $5 \mathrm{mM}$ EDTA). Lysates were subjected to two rounds of centrifugation at $13,200 \mathrm{rpm}$ for $15 \mathrm{~min}$ each at $4{ }^{\circ} \mathrm{C}$ in a benchtop centrifuge. Supernatants were incubated with monoclonal mouse anti-SPO11 antibody $180(3 \mu \mathrm{g}$ per pair of testes) at $4{ }^{\circ} \mathrm{C}$ for $1 \mathrm{~h}$, followed by addition of $40 \mu \mathrm{l}$ Protein AAgarose beads (Roche) and incubated for an additional $3 \mathrm{~h}$ at $4{ }^{\circ} \mathrm{C}$. Beads were washed three times with IP buffer $(1 \%$ Triton X-100, $150 \mathrm{mM} \mathrm{NaCl}, 15 \mathrm{mM}$ Tris- $\mathrm{HCl}$ at $\mathrm{pH} 8.0$, $1 \mathrm{mM}$ EDTA). Immunoprecipitates were eluted with Laemmli sample buffer. Samples were fractionated on $8 \%$ SDS-PAGE and transferred to a PVDF membrane by semidry transfer system (Bio-Rad). For Western analysis, membranes were probed with antibody anti-mSPO11 antibody 180 (1:2,000 in PBS containing 0.1\% Tween 20 and $5 \%$ non-fat dry milk) overnight at $4{ }^{\circ} \mathrm{C}$ and then with horseradish peroxidase-conjugated protein A (Abcam; 1:10,000 in PBS containing $0.1 \%$ Tween 20 and $5 \%$ non-fat dry milk) for $2 \mathrm{~h}$ at room temperature. Signals were detected using the $\mathrm{ECL}+$ reagent (GE Healthcare).

Acknowledgments We thank Cristina Antinozzi and Simone Balzano (University of Rome Tor Vergata) for their contribution to mice genotyping and colony maintenance during the early stages of the project; Julian Lange (Memorial Sloan-Kettering Cancer Center-MSKCC) for his advices with respect to SPO11 immunoprecipitations; Flavia Botti (University of Rome Tor Vergata) for technical assistance in the preparation of sample for the histological analyses; Maria Jasin and Scott Keeney (MSKCC) for providing Spo11 mice; and Kristina Havas-Cavaletti (EMBL) for editing the manuscript.

\section{Compliance with ethical standards}

Conflict of Interest The authors declare that they have no competing interests.

Ethical approval All applicable international, national, and/or institutional guidelines for the care and use of animals were followed. This article does not contain any studies with human participants performed by any of the authors.

Informed consent Informed consent was obtained from all individual participants included in the study.

Authors' contributions MB conceived and coordinated the study. MF, SDC, DGdR, AL, MM, MDG, SDS, and MB designed and performed the experiments. MP contributed reagents/materials/analysis tools. MF and MB wrote the paper. DGdR, MDG, MP, and PR drafted the article or revisited it critically. 
Funding This work was supported by Telethon grant no. GGP12189 to Marco Barchi and Claudio Sette.

Open Access This article is distributed under the terms of the Creative Commons Attribution 4.0 International License (http:// creativecommons.org/licenses/by/4.0/), which permits unrestricted use, distribution, and reproduction in any medium, provided you give appropriate credit to the original author(s) and the source, provide a link to the Creative Commons license, and indicate if changes were made.

\section{References}

Ahmed EA, de Rooij DG (2009) Staging of mouse seminiferous tubule cross-sections. Methods Mol Biol 558:263-277

Argunhan B, Farmer S, Leung WK, Terentyev Y, Humphryes N, Tsubouchi T, Toyoizumi H, Tsubouchi H (2013) Direct and indirect control of the initiation of meiotic recombination by DNA damage checkpoint mechanisms in budding yeast. PLoS One 8(6), e65875

Barchi M, Mahadevaiah S, Di Giacomo M, Baudat F, de Rooij DG, Burgoyne PS, Jasin M, Keeney S (2005) Surveillance of different recombination defects in mouse spermatocytes yields distinct responses despite elimination at an identical developmental stage. Mol Cell Biol 25(16):7203-7215

Barchi M, Roig I, Di Giacomo M, de Rooij DG, Keeney S, Jasin M (2008) ATM promotes the obligate XY crossover and both crossover control and chromosome axis integrity on autosomes. PLoS Genet 4(5), e1000076

Barchi M, Geremia R, Magliozzi R, Bianchi E (2009) Isolation and analyses of enriched populations of male mouse germ cells by sedimentation velocity: the centrifugal elutriation. Methods Mol Biol 558: 299-321

Baudat F, Manova K, Yuen JP, Jasin M, Keeney S (2000) Chromosome synapsis defects and sexually dimorphic meiotic progression in mice lacking Spo11. Mol Cell 6(5):989-998

Bellani MA, Romanienko PJ, Cairatti DA, Camerini-Otero RD (2005) SPO11 is required for sex-body formation, and Spo11 heterozygosity rescues the prophase arrest of Atm-/- spermatocytes. J Cell Sci 118(Pt 15):3233-3245

Bellani MA, Boateng KA, McLeod D, Camerini-Otero RD (2010) The expression profile of the major mouse SPO11 isoforms indicates that SPO11beta introduces double strand breaks and suggests that SPO11alpha has an additional role in prophase in both spermatocytes and oocytes. Mol Cell Biol 30(18): $4391-4403$

Bishop DK (1994) RecA homologs Dmc1 and Rad51 interact to form multiple nuclear complexes prior to meiotic chromosome synapsis. Cell 79(6):1081-1092

Cole F, Kauppi L, Lange J, Roig I, Wang R, Keeney S, Jasin M (2012) Homeostatic control of recombination is implemented progressively in mouse meiosis. Nat Cell Biol 14(4):424-430

Cooper TJ, Wardell K, Garcia V, Neale MJ (2014) Homeostatic regulation of meiotic DSB formation by ATM/ATR. Exp Cell Res 329(1): 124-131

Davis L, Barbera M, McDonnell A, McIntyre K, Sternglanz R, Jin Q, Loidl J, Engebrecht J (2001) The Saccharomyces cerevisiae MUM2 gene interacts with the DNA replication machinery and is required for meiotic levels of double strand breaks. Genetics 157(3): $1179-1189$

de Rooij DG, de Boer P (2003) Specific arrests of spermatogenesis in genetically modified and mutant mice. Cytogenet Genome Res 103(3-4):267-276
Difilippantonio S, Celeste A, Fernandez-Capetillo O, Chen HT, Reina San Martin B, Van Laethem F, Yang YP, Petukhova GV, Eckhaus M, Feigenbaum L, Manova K, Kruhlak M, Camerini-Otero RD, Sharan S, Nussenzweig M, Nussenzweig A (2005) Role of Nbs1 in the activation of the Atm kinase revealed in humanized mouse models. Nat Cell Biol 7(7):675-685

Eaker S, Cobb J, Pyle A, Handel MA (2002) Meiotic prophase abnormalities and metaphase cell death in MLH1-deficient mouse spermatocytes: insights into regulation of spermatogenic progress. Dev Biol 249(1):85-95

Gray S, Allison RM, Garcia V, Goldman AS, Neale MJ (2013) Positive regulation of meiotic DNA double-strand break formation by activation of the DNA damage checkpoint kinase Mec1(ATR). Open Biol 3(7):130019

Hamer G, Novak I, Kouznetsova A, Hoog C (2008) Disruption of pairing and synapsis of chromosomes causes stage-specific apoptosis of male meiotic cells. Theriogenology 69(3):333-339

Henderson KA, Keeney S (2004) Tying synaptonemal complex initiation to the formation and programmed repair of DNA double-strand breaks. Proc Natl Acad Sci U S A 101(13):4519-4524

Joshi N, Brown MS, Bishop DK, Borner GV (2015) Gradual implementation of the meiotic recombination program via checkpoint pathways controlled by global DSB levels. Mol Cell 57(5):797-811

Kauppi L, Barchi M, Baudat F, Romanienko PJ, Keeney S, Jasin M (2011) Distinct properties of the XY pseudoautosomal region crucial for male meiosis. Science 331(6019):916-920

Kauppi L, Barchi M, Lange J, Baudat F, Jasin M, Keeney S (2013a) Numerical constraints and feedback control of double-strand breaks in mouse meiosis. Genes Dev 27(8):873-886

Kauppi L, Jasin M, Keeney S (2013b) How much is enough? Control of DNA double-strand break numbers in mouse meiosis. Cell Cycle 12(17):2719-2720

Keeney S (2001) Mechanism and control of meiotic recombination initiation. Curr Top Dev Biol 52:1-53

Keeney S (2008) Spo11 and the formation of DNA double-strand breaks in meiosis. Genome Dyn Stab 2:81-123

Keeney S, Baudat F, Angeles M, Zhou ZH, Copeland NG, Jenkins NA, Manova K, Jasin M (1999) A mouse homolog of the Saccharomyces cerevisiae meiotic recombination DNA transesterase Spo11p. Genomics 61(2):170-182

Keeney S, Lange J, Mohibullah N (2014) Self-organization of meiotic recombination initiation: general principles and molecular pathways. Annu Rev Genet 48:187-214

Lange J, Pan J, Cole F, Thelen MP, Jasin M, Keeney S (2011) ATM controls meiotic double-strand-break formation. Nature 479(7372): 237-240

Lenzi ML, Smith J, Snowden T, Kim M, Fishel R, Poulos BK, Cohen PE (2005) Extreme heterogeneity in the molecular events leading to the establishment of chiasmata during meiosis I in human oocytes. Am J Hum Genet 76(1):112-127

Lipkin SM, Moens PB, Wang V, Lenzi M, Shanmugarajah D, Gilgeous A, Thomas J, Cheng J, Touchman JW, Green ED, Schwartzberg P, Collins FS, Cohen PE (2002) Meiotic arrest and aneuploidy in MLH3-deficient mice. Nat Genet 31(4):385-390

Mahadevaiah SK, Bourc'his D, de Rooij DG, Bestor TH, Turner JM, Burgoyne PS (2008) Extensive meiotic asynapsis in mice antagonises meiotic silencing of unsynapsed chromatin and consequently disrupts meiotic sex chromosome inactivation. J Cell Biol 182(2):263-276

Muciaccia B, Boitani C, Berloco BP, Nudo F, Spadetta G, Stefanini M, de Rooij DG, Vicini E (2013) Novel stage classification of human spermatogenesis based on acrosome development. Biol Reprod 89(3):60 
Neale MJ, Pan J, Keeney S (2005) Endonucleolytic processing of covalent protein-linked DNA double-strand breaks. Nature 436(7053): 1053-1057

Pacheco S, Marcet-Ortega M, Lange J, Jasin M, Keeney S, Roig I (2015) The ATM signaling cascade promotes recombination-dependent pachytene arrest in mouse spermatocytes. PLoS Genet 11(3), e1005017

Page SL, Hawley RS (2004) The genetics and molecular biology of the synaptonemal complex. Annu Rev Cell Dev Biol 20:525-558

Pellegrini M, Claps G, Orlova VV, Barrios F, Dolci S, Geremia R, Rossi P, Rossi G, Arnold B, Chavakis T, Feigenbaum L, Sharan SK, Nussenzweig A (2011) Targeted JAM-C deletion in germ cells by Spo11-controlled Cre recombinase. J Cell Sci 124(Pt 1):91-99

Roig I, Dowdle JA, Toth A, de Rooij DG, Jasin M, Keeney S (2010) Mouse TRIP13/PCH2 is required for recombination and normal higher-order chromosome structure during meiosis. PLoS Genet 6(8)
Romanienko PJ, Camerini-Otero RD (1999) Cloning, characterization, and localization of mouse and human SPO11. Genomics 61(2): $156-169$

Romanienko PJ, Camerini-Otero RD (2000) The mouse Spo11 gene is required for meiotic chromosome synapsis. Mol Cell 6(5):975-987

Russell LE, Hikim RA, Clegg APS (eds) (1990) Histological and histopatological evaluation of the testis. Cache River Press, Clerwater

Storlazzi A, Gargano S, Ruprich-Robert G, Falque M, David M, Kleckner N, Zickler D (2010) Recombination proteins mediate meiotic spatial chromosome organization and pairing. Cell 141(1):94-106

Terasawa M, Shinohara A, Hotta Y, Ogawa H, Ogawa T (1995) Localization of RecA-like recombination proteins on chromosomes of the lily at various meiotic stages. Genes Dev 9(8):925-934

Tesse S, Storlazzi A, Kleckner N, Gargano S, Zickler D (2003) Localization and roles of Ski8p protein in Sordaria meiosis and delineation of three mechanistically distinct steps of meiotic homo$\log$ juxtaposition. Proc Natl Acad Sci U S A 100(22):12865-12870 